\title{
The correlation analysis between the Oxford classification of Chinese IgA nephropathy children and renal outcome - a retrospective cohort study
}

Heyan Wu ${ }^{1,2}$, Zhengkun Xia ${ }^{1 *} \mathbb{D}$, Chunlin Gao ${ }^{1 *}$, Pei Zhang ${ }^{1}$, Xiao Yang ${ }^{1}$, Ren Wang ${ }^{3}$, Meiqiu Wang ${ }^{1}$ and Yingchao Peng ${ }^{1}$

\begin{abstract}
Background: The 2016 Oxford Classification's MEST-C scoring system predicts outcomes in adults with IgA nephropathy (IgAN), but it lacks tremendous cohort validation in children with IgAN in China. We sought to verify whether the Oxford classification could be used to predict the renal outcome of children with IgAN.

Methods: In this retrospective cohort study, 1243 Chinese IgAN children who underwent renal biopsy in Jinling Hospital were enregistered from 2000 to 2017. The combined endpoint was defined as either a $\geq 50 \%$ reduction in estimated glomerular filtration rate (eGFR) or end-stage renal disease (ESRD). We probed into the relevance betwixt the Oxford classification and renal prognosis.

Results: There were 29\% of children with mesangial proliferation(M1), 35\% with endocapillary proliferation (E1), $37 \%$ with segmental sclerosis/adhesion lesion (S1), 23\% with moderate tubular atrophy/interstitial fibrosis (T1 25-50\% of cortical area involved), 4.3\% with severe tubular atrophy/interstitial fibrosis (T2 > 50\% of cortical area involved), 44\% with crescent in $<25 \%$ of glomeruli(C1), and $4.6 \%$ with crescent in $>25 \%$ of glomeruli (C2). All children were followed for a medial of $7.2(4.6-11.7)$ years, 171 children (14\%) arrived at the combined endpoint. The multivariate COX regression model revealed that the presence of lesions $S(H R 2.7,95 \% \mathrm{Cl} 1.8 \sim 4.2, P<0.001)$ and $\mathrm{T}(\mathrm{HR} 6.6,95 \% \mathrm{Cl} 3.9 \sim 11.3$, $P<0.001)$ may be the reason for poorer prognosis in the whole cohort. In contrast, $C$ lesion showed a significant association with the outcome only in children received no immunosuppressive treatment.
\end{abstract}

Conclusions: This study revealed that $\mathrm{S}$ and $\mathrm{T}$ lesions were useful as the long-term renal prognostic factors among Chinese IgAN children.

Keywords: IgA nephropathy, Oxford classification, Children, Renal outcome

\footnotetext{
* Correspondence: njxzk@126.com; shuangmu34@163.com

'Department of Pediatrics, Jinling Hospital, The First School of Clinical

Medicine, Southern Medical University, Nanjing, China

Full list of author information is available at the end of the article
}

(C) The Author(s). 2020 Open Access This article is licensed under a Creative Commons Attribution 4.0 International License, which permits use, sharing, adaptation, distribution and reproduction in any medium or format, as long as you give appropriate credit to the original author(s) and the source, provide a link to the Creative Commons licence, and indicate if changes were made. The images or other third party material in this article are included in the article's Creative Commons licence, unless indicated otherwise in a credit line to the material. If material is not included in the article's Creative Commons licence and your intended use is not permitted by statutory regulation or exceeds the permitted use, you will need to obtain permission directly from the copyright holder. To view a copy of this licence, visit http://creativecommons.org/licenses/by/4.0/. The Creative Commons Public Domain Dedication waiver (http://creativecommons.org/publicdomain/zero/1.0/) applies to the data made available in this article, unless otherwise stated in a credit line to the data. 


\section{Background}

IgA nephropathy (IgAN), being particularly frequent in Asia, is the primary reason for end-stage renal disease (ESRD) in all ages [1]. Since IgAN does not exhibit a specific serologic profile, a percutaneous kidney biopsy remains a definitive tool to establish the diagnosis of IgAN [2]. Additionally, the prognostic value of histological data has become increasingly recognized in the past decade [3]. As a new pathological classification standard to judge the renal prognosis of IgAN, the Oxford classification [4] has been put forward in recent years. The purpose of the Oxford classification was to consider the pathological features associated with clinical outcomes independently of clinical data and to improve the current ability to predict outcomes in IgAN patients. Although the classification standard has been formulated by rigorous methodology, its clinical application in children needs to be further verified. What's more, IgAN has regional and ethnic differences, which determines that the Oxford classification needs to be refined in different populations and races. In this study, the clinical and pathological data of IgAN followed up for a long time in our department were retrospectively analyzed to assess the predictability of Oxford classification among Chinese children.

\section{Methods}

\section{Inclusion criteria and clinical data set}

Children with IgAN who underwent renal biopsy in Jinling Hospital were enrolled from January 1, 2000, to December 31,2017 , in this retrospective cohort study. The inclusion criteria were follow-up $\geq 12$ months, age at the time of biopsy $\leq 18$ years, a minimum of eight glomeruli and an initial eGFR $\geq 15 \mathrm{ml} / \mathrm{min} / 1.73 \mathrm{~m}^{2}$. The exclusion criteria were secondary IgAN caused by Henoch-Schonlein purpura, liver cirrhosis, systemic lupus erythematosus, hepatitis B virus infection, tumour, ankylosing spondylitis and psoriasis. The following clinical data including age, gender, duration from onset to renal biopsy, estimate glomerular filtration rate (eGFR), mean arterial pressure (MAP), proteinuria and therapeutic regimen [Renin angiotensin aldosterone system blockade (RASB), glucocorticoid (GC) and other immunosuppressant agents] were collected during biopsy and follow-up. Immunosuppressive therapy after kidney biopsy was defined as treatment with any immunosuppressive agent, regardless of duration or dose.

\section{Definitions}

eGFR was calculated using the Schwartz formula [5], and the CKD-EPI equation [6] was used in adolescents aged $>16$ years at the time of biopsy. ESRD was defined as eGFR $<15 \mathrm{~mL} / \mathrm{min} / 1.73 \mathrm{~m}^{2}$, initiation of dialysis or transplantation. Survival time was defined from the time of biopsy until the date of the last follow-up, the incidence of the event of interest, or March 2019 (end of the study). The combined endpoint was defined as either $\geq 50 \%$ reduction eGFR or ESRD or death.

\section{Pathology review}

Renal pathology was scored according to the Oxford classification of IgAN [4], assessed by the following parameters: mesangial hypercellularity $(\mathrm{M})$, scored as absent (M0) or (M1) if $\geq 50 \%$ of glomeruli had more than three cells per mesangial area; endocapillary hypercellularity (E), scored as E0 if absent or E1 if present; segmental glomerulosclerosis or adhesion (S), scored (S0) if absent or (S1) if present; tubular atrophy/interstitial fibrosis(T), scored as T0 (0-25\% of cortical area), T1 (26-50\% of cortical area), or $\mathrm{T} 2(>50 \%$ of cortical area); cellular/ fibrocellular crescents scored as C0 (no crescents), $\mathrm{C} 1$ (crescents in at least one but $<25 \%$ of glomeruli), or C2 (crescents in more than $25 \%$ of glomeruli). Immunofluorescence studies were performed (IgG, IgA, IgM, C3) and showed at least 1+ (on scale from 0 to $3+$ ) mesangial deposition of IgA, with IgA being the dominant immunoglobulin deposited in the glomeruli. The intensity of deposits as determined via immunofluorescence microscopy was graded semi-quantitatively on a scale from 0 to $3+$, where $0=$ no, $1+=$ slight, $2+=$ moderate, and $3+=$ intense. At least two pathologists blinded to patient outcomes at the time of review confirmed the pathological results.

\section{Statistical analyses}

Continuous variables were presented as mean \pm standard deviation (normally distributed variables) or median with interquartile range (IQR, non-normally distributed variables) for data distribution. Categorical variables are presented as percentages. The Kruskal-Wallis test and chi-squared test were used to compare continuous and categorical variables, respectively. The Kaplan-Meier curve was used to illustrate univariate differences between groups of pathological variables, and the log-rank test were used to test the two curves differences. The Cox proportional hazards regression model was conducted on univariable and multivariable analyses of Oxford classification in the IgAN children. Univariable Cox regression analysis was performed for each pathological variable. Multivariable Cox regression analysis (Backward: LR approach) adjusting for other clinically essential factors including initial eGFR, initial mean arterial pressure, and initial proteinuria was performed to appraise the function of MEST-C scoring system on the renal outcome. Hazard ratio (HR) with $95 \%$ confidence interval $(\mathrm{CI})$ for each variable was estimated. All probabilities were two-tailed, and $P$-value $<0.05$ was considered statistically significant. 
Data analysis was executed using SPSS for windows version 26 (IBM Corporation, Armonk, NY).

\section{Results \\ Clinical features}

We enrolled 1243 Chinese children diagnosed with primary IgAN from 2000 to 2017 in Jinling Hospital. Table 1 outlines the clinical, treatment and follow-up characteristics of the study children. The average age of children at diagnosis was $14 \pm 4$ years, with maledominated (68\%). The average value of MBP was $89 \pm$ $16 \mathrm{mmHg}$, initial proteinuria was 0.6 (interquartile ranges, $0.3-1.4) \mathrm{g} /$ day per $1.73 \mathrm{~m}^{2}$, and the initial eGFR was $102 \pm 20 \mathrm{ml} / \mathrm{min}$ per $1.73 \mathrm{~m}^{2}$. All children were followed for a medial of $7.2(4.6-11.7)$ years, 171 children (14\%) arrived at combined endpoint (ESRD, $n=82$; $\geq 50 \%$ eGFR decline, $n=89$ ). During the follow-up period, $70 \%$ of children were treated with RASB, $45 \%$ were treated with GC, and 19\% received GC combined other immunosuppressive drugs.

\section{Pathological findings}

The average of glomeruli was $20.4 \pm 4.7$ per biopsy. Based on Oxford classification,29\% of the children showed M1, 35\% showed E1, 37\% showed S1, 23\% showed T1, 4.3\% showed T2, 44\% showed C1 and 4.6\% showed C2 (Table 2). The distribution of the percentage of crescents observed in every child was shown in Fig. 1. Of $48.6 \%$ children with any cellular/ fibrocellular crescents, $28 \%$ had crescents in $<10 \%$ of glomeruli, whereas

Table 1 Baseline and follow-up characteristics ( $n=1243)$

\begin{tabular}{ll}
\hline At renal biopsy & Values at renal biopsy \\
\hline Male, \% & 68 \\
Age, years & $14 \pm 4$ \\
Duration from onset to renal biopsy (months) & $12.0(0.8,96.5)$ \\
eGFR, ml/min per $1.73 \mathrm{~m}^{2}$ & $102 \pm 20$ \\
MAP, mmHg & $89 \pm 16$ \\
Proteinuria, g/day per 1.73m & $0.6(0.3-1.4)$ \\
Follow-up parameters & Values during follow-up \\
Length of follow-up, years & $7.2(4.6-11.7)$ \\
RASB, \% & 70 \\
Any immunosuppression, \% & 64 \\
GC, \% & 45 \\
GC + IS, \% & 19 \\
Combined event, \% & 14 \\
ESRD, \% & 6.6 \\
$50 \%$ reduction in initial eGFR, $\%$ & 7.2
\end{tabular}

Values are expressed as mean $\pm \mathrm{SD}$; medians (interquartile ranges), or percentages

Abbreviations: eGFR Estimate glomerular filtration rate, MAP Mean arterial pressure, RASB Renin angiotensin aldosterone system blockade, GC

Glucocorticoid, IS Other immunosuppressant, ESRD End-stage of renal disease
Table 2 Pathological findings at the time of biopsy in children with IgA nephropathy $(n=1243)$

\begin{tabular}{ll}
\hline Pathology findings & Values at renal biopsy \\
\hline The number of glomeruli per biopsy & $20.4 \pm 4.7$ \\
MEST-C score & $\%$ of total biopsies \\
M1 & 29 \\
E1 & 35 \\
S1 & 37 \\
T1 & 23 \\
T2 & 4.3 \\
C1 & 44 \\
C2 & 4.6 \\
Deposition site of immunoglobulins & $\%$ of total biopsies \\
Pure-mesangium & 68 \\
Mesangium+ capillary loop & 32 \\
Immunoglobulins deposits & $\%$ of total biopsies \\
Glomerular lgG deposition & 25 \\
Glomerular lgM deposition & 44 \\
Glomerular C3 deposition & 84 \\
Glomerular C4 deposition & 1.1 \\
Intensity of IgA deposits ${ }^{\text {a }}$ & $\%$ of total biopsies \\
1+ & 5.6 \\
2+ & 13 \\
$3+$ & 81 \\
\hline Val are expressed as mean 5 or number &
\end{tabular}

Values are expressed as mean \pm SD or number (percentage); The intensity of $\operatorname{IgA}$ deposits as determined via immunofluorescence microscopy was graded semi-quantitatively on a scale from 0 to $3+$, where 0 no, $1+=$ slight, $2+=$ moderate, and $3+=$ intense

Abbreviations: M1 Mesangial hypercellularity $>0.5, E 1$ Presence of endocapillary hypercellularity, S1 Presence of segmental glomerulosclerosis, $T 1$ Tubular atrophy/ interstitial fibrosis $26-50 \%$ of cortical area, $T 2$ Tubular atrophy/ interstitial fibrosis $\geq 50 \%$ of cortical area, C1 Crescents in at least one but $<25 \%$ of glomeruli, C2 Crescents in more than $25 \%$ of glomeruli

9.4\% had a fraction of glomeruli with crescents onetenth or more, $6.6 \%$ had a fraction of glomeruli with crescents one-sixth or more, and only $4.6 \%$ had a fraction of glomeruli with crescents one-fourth or more. The percentage of immunoglobulins deposited only in the mesangial region was $68 \%$, while $32 \%$ of immunoglobulins were deposited in both the mesangial and capillary loop regions. $25 \%$ of children showed positive glomerular staining for IgG, $44 \%$ showed positive glomerular staining for IgM, $84 \%$ showed positive glomerular staining for $\mathrm{C} 3$, and $1.1 \%$ showed positive glomerular staining for $\mathrm{C} 4$. The immunofluorescence intensity of IgA was between $1+$ and $3+$, including $5.6 \%$ of $1+, 13 \%$ of $2+$ and $81 \%$ of $3+$.

Effects of different kidney biopsy time on the variables in Oxford classification

The median time (12 months) of onset to renal biopsy was selected as the cut-off point to analyze the effect of 


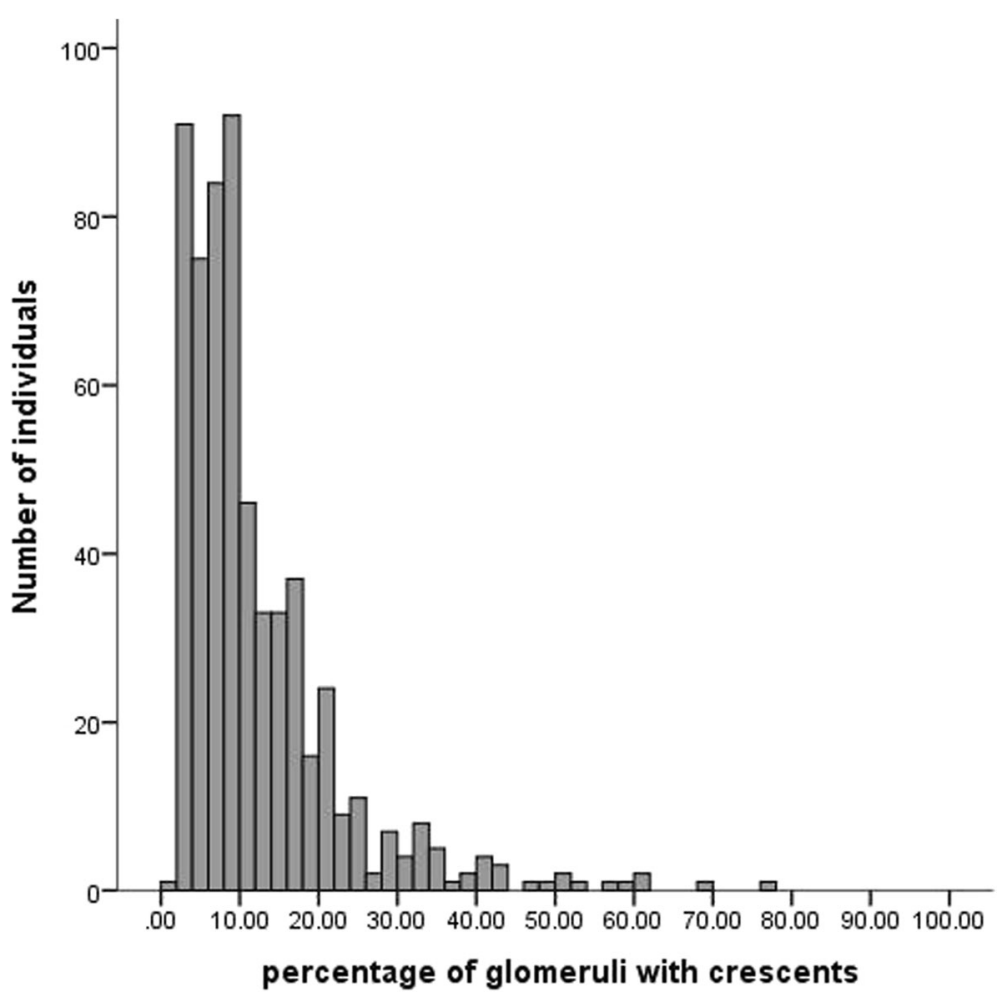

Fig. 1 Distribution of the percentage of glomeruli with crescents in biopsies with any crescents. Crescents were present in 599(48\%) of 1243 total biopsies

biopsy time on variables in the Oxford classification From Table 3. It showed that when the time of onset to renal biopsy was less than 12 months, the patient's lesions were milder, dominated by $\mathrm{S} 0\left(x^{2}=354.5, P<0.001\right)$, T0 $\left(x^{2}=323.3, P<0.001\right)$, and $\mathrm{C} 0\left(\chi^{2}=437.6, P<0.001\right)$. On the contrary, when the time of onset to renal biopsy was longer than 12 months, the lesions were corrected mainly by $\mathrm{S} 1, \mathrm{~T} 1-2$ and $\mathrm{C} 1-2$. Concerning $\mathrm{E}$ and $\mathrm{M}$

Table 3 Comparison of all kinds of lesions with different time of onset to renal biopsy $(n=1243)$

\begin{tabular}{|c|c|c|c|c|}
\hline \multirow[t]{2}{*}{ Variables } & \multicolumn{2}{|c|}{ Time from onset to renal biopsy } & \multirow[t]{2}{*}{$x^{2}$} & \multirow[t]{2}{*}{$P$-value } \\
\hline & $\leq 12$ months & $>12$ months & & \\
\hline $\mathrm{M} 0 / \mathrm{M} 1$ & $449 / 172$ & $433 / 189$ & 1.1 & 0.296 \\
\hline $\mathrm{E} 0 / \mathrm{E} 1$ & $411 / 210$ & $401 / 221$ & 0.4 & 0.525 \\
\hline So/ S1 & $554 / 67$ & $235 / 387$ & 354.5 & $<0.001$ \\
\hline $\mathrm{T} 0 / \mathrm{T} 1 / \mathrm{T} 2$ & $595 / 21 / 5$ & $315 / 259 / 48$ & 323.3 & $<0.001$ \\
\hline $\mathrm{CO} / \mathrm{C} 1 / \mathrm{C} 2$ & $506 / 104 / 11$ & $138 / 438 / 46$ & 437.6 & $<0.001$ \\
\hline
\end{tabular}

Abbreviations: M0 Mesangial hypercellularity $\leq 0.5$, M1 Mesangial hypercellularity $>0.5, E 0$ Absence of endocapillary hypercellularity, E1 Presence of endocapillary hypercellularity; SO absence of segmental glomerulosclerosis, S1 Presence of segmental glomerulosclerosis, TO Tubular atrophy/ interstitial fibrosis $0-25 \%$ of cortical area, $T 1$ Tubular atrophy/ interstitial fibrosis $26-50 \%$ of cortical area, T2 Tubular atrophy/interstitial fibrosis $\geq 50 \%$ of cortical area, CO Absence of crescents, C1 Crescents in at least one but $<25 \%$ of glomeruli, C2 Crescents in more than $25 \%$ of glomeruli lesions, there was no significant difference in time from onset to renal biopsy within available data.

\section{Associations between clinical and histologic variables}

Linear regression analysis of Oxford classification with the most robust indicators for estimating renal decline (eGFR, MAP and proteinuria) was performed to explore the correlation between Oxford classification and clinical signs. As shown in Table 4, Children with S1, T1-2 and C1-2 lesions were associated with a reduced initial eGFR at the time of biopsy. All histological lesions (M1, E1, S1, T1-2, and C1-2) were associated individually with higher initial proteinuria at the time of biopsy. All histological lesions were associated with more upper initial MAP at the time of biopsy.

\section{Renal survival IgAN children according to Oxford classification}

As presented in Fig. 2, the Kaplan-Meier analyses revealed that lesion S (Log-Rank, $X^{2}=14.796, P<0.001$; Fig. $2 c)$ and $\mathrm{T}\left(X^{2}=48.976, P<0.001\right.$; Fig. $\left.2 d\right)$ were each correlation with renal survival. However, lesions $M\left(x^{2}=\right.$ 1.459, $P=0.177$, Fig. 2a), E $\left(\chi^{2}=2.399, P=0.331\right.$, Fig. $\left.2 \mathrm{~b}\right)$ and $C\left(X^{2}=6.218, P=0.054\right.$, Fig. $\left.2 \mathrm{e}\right)$ were not associated with renal outcome. 
Table 4 Linear Regression Analysis of Oxford Classification and Clinical Indicators at Renal Biopsy

\begin{tabular}{|c|c|c|c|c|c|c|}
\hline \multirow{2}{*}{$\begin{array}{l}\text { Clinical } \\
\text { indicators }\end{array}$} & \multicolumn{2}{|c|}{$\operatorname{MAP}(\mathrm{mmHg})$} & \multicolumn{2}{|c|}{ eGFR $\left(\mathrm{ml} / \mathrm{min} / 1.73 \mathrm{~m}^{2}\right)$} & \multicolumn{2}{|c|}{ Proteinuria (g/day/1.73m²) } \\
\hline & $\mathrm{R}$ & $P$-value & $\mathrm{R}$ & $P$-value & $\mathrm{R}$ & $P$-value \\
\hline$\overline{M 1}$ & 0.342 & $<0.001$ & 0.044 & 0.133 & 0.569 & $<0.001$ \\
\hline E1 & 0.338 & $<0.001$ & -0.331 & 0.389 & 0.527 & $<0.001$ \\
\hline S1 & 0.541 & 0.042 & -0.744 & 0.007 & 0.604 & $<0.001$ \\
\hline $\mathrm{T} 1-2$ & 0.532 & $<0.001$ & -0.578 & $<0.001$ & 0.689 & $<0.001$ \\
\hline $\mathrm{C} 1-2$ & 0.549 & 0.008 & -0.447 & $<0.001$ & 0.447 & $<0.001$ \\
\hline
\end{tabular}

Linear Regression results are results from separate models for each independent variable

Abbreviations: MAP Mean arterial blood pressure, eGFR Estimate glomerular filtration rate, M1 Mesangial hypercellularity $>0.5$, E1 Presence of endocapillary hypercellularity, S1 Presence of segmental glomerulosclerosis, T1 Tubular atrophy/ interstitial fibrosis 26-50\% of cortical area, T2 Tubular atrophy/interstitial fibrosis $\geq 50 \%$ of cortical area, C1 Crescents in at least one but $<25 \%$ of glomeruli, C2 Crescents in more than $25 \%$ of glomeruli

\section{Associations between pathologic features and renal outcome}

The associations between pathological features and renal outcomes were analyzed in a COX regression model (Table 5). With univariate COX regression model, renal outcome from a combined event were both associated significantly with lesions S (HR3.5, 95\%CI $2.3 \sim 5.3, P<$ 0.001), T (HR 2.6, 95\%CI $2.1 \sim 3.3, P<0.001)$ and $\mathrm{C}(\mathrm{HR}$ 2.1, 95\%CI $1.5 \sim 2.8, P<0.001)$. However, the lesion of $\mathrm{M}$ (HR 1.8, 95\%CI 1.3 2.3, $P=0.115$ ), and E (HR 1.4, $95 \%$ CI $0.9 \sim 2.1, P=0.326)$ were not associated with renal outcome. When adjusted for clinically essential data in the multivariate COX regression model, only $\mathrm{S}$ (HR2.7, 95\%CI1.8 4.2, $P<0.001$ ) and T (HR6.6, 95\%CI $3.9 \sim 11.3, \quad P<0.001)$ lesions remained as independent predictors of renal outcome.

\section{Predictive value of lesion $M, E$ and $C$ between immunosuppressive and without immunosuppressive groups}

We further assessed the predictive value of lesions $\mathrm{M}, \mathrm{E}$ and $C$ in children who received no immunosuppressive treatment to assess their natural predictive value. Children with crescent who didn't receive immunosuppressive therapy experienced worse survival from the combined event (Fig. 3e), but this difference disappeared after received immunosuppression (Fig. 3f). The predictive value of lesion $M$ and $E$ were not changed by adding immunosuppressive treatment (Fig. 3a-d).

\section{Discussion}

This study investigated the clinical and histopathologic predictors of poor prognosis in pediatric patients with IgAN. The median duration from onset to renal biopsy was 12 months in our cohort. An early diagnosis seemed to be the primary reason for a low frequency of chronic and severe lesions such as lesion $\mathrm{S}, \mathrm{T}$ and $\mathrm{C}$. In our cohort, we confirmed that $\mathrm{S}$ and $\mathrm{T}$ were independent risk factors associated with renal outcomes. The lesion $\mathrm{C}$ enhanced the ability to predict progression only in those who did not receive immunosuppression. Lesion $M$ and
E were not significant variables, which may weaken their predictive values because of the low percentage in the cohort. The independent predictive value of pathology MEST-C score was reduced by immunosuppressive therapy.

Our results suggested that patients with severe pathological lesions (e.g. S、T、C) were associated with lower eGFR, higher blood pressure and higher proteinuria, which were consistent with other findings [7-9]. Glomerular hypertension may mediate progressive renal damage by leading to glomerular hyperfiltration and glomerular enlargement [7]. For the control target of blood pressure in IgAN, the KDIGO guidelines [8] pointed out that when proteinuria $>0.3 \mathrm{~g} / \mathrm{day}$, the recommended target blood pressure (BP) was $<130 / 80 \mathrm{mmHg}$, and when proteinuria $>1 \mathrm{~g} / \mathrm{d}$, the recommended target $\mathrm{BP}$ was $<125 / 75 \mathrm{mmHg}$. Bellur et al. [9] showed that $\mathrm{S}$ was strongly associated with proteinuria and lower eGFR levels, which was consistent with our conclusion. Previous studies [10] have shown that $\mathrm{T}$ was an independent risk factor for poor renal prognosis and associated with BP. Some scholars $[11,12]$ had found that the level of eGFR was lower in patients with IgAN with extensive crescent formation, and there was a negative correlation between eGFR and the proportion of crescents. Thus, it can be concluded that the most critical risk factors for the progression of IgAN (proteinuria, eGFR, MAP) are significantly correlated with the pathological damage found by renal biopsy, which reflects the value of the combination of clinical and pathological risk factors in judging the prognosis of IgAN children.

The lesion $M$ was not a significant risk for renal outcome in our cohort. We speculated that it might be difficult to address its value because of its low prevalence in our study. But the value of lesion $\mathrm{M}$ as an independent risk for progression is debated. On the one hand, the VALIGA cohort [13] and a Chinese adult cohort [14] confirmed $\mathrm{M}$ lesion as a significant factor for progression, but on the other Shima et al. [15] reported that M had lost predictive value in patients receiving immunosuppressive therapy. By and large, the presence of $M$ 


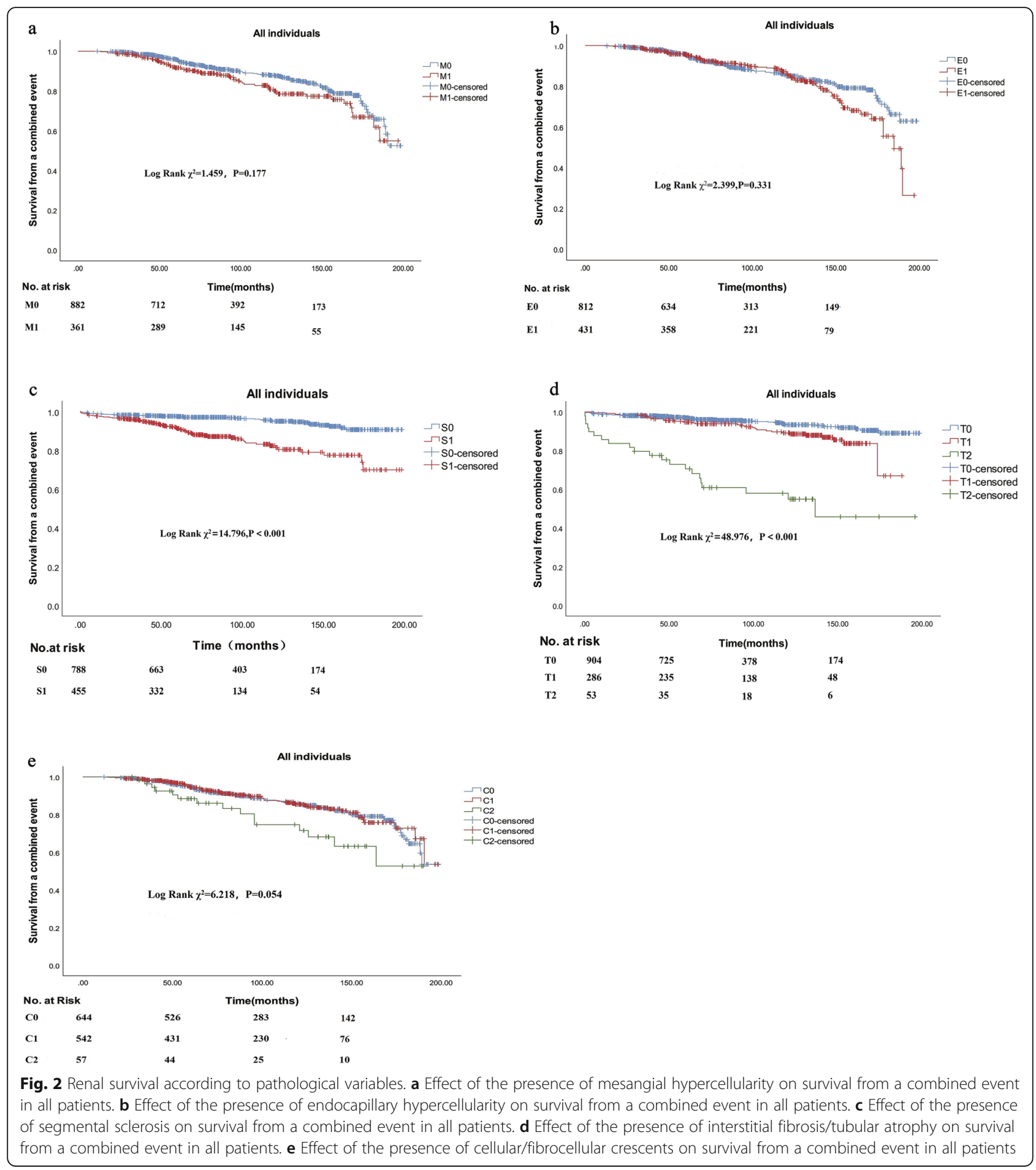

lesion appears to have a negligible correlation with renal outcomes.

The lesion E, observed in 35\% of the children, did not independently predict clinical outcome in the whole cohort. This was highly consistent with the findings in the Oxford classification cohort [16]. However, two studies in which patients did not receive immunosuppressive therapy $[17,18]$ reported that E1 was independently associated with more rapid loss of renal function and worse renal survival, which indirectly suggested that proliferative lesions were treatment-responsive. We conjectured that the widespread use of immunosuppressants might have influenced the absence of correlation between E lesion and outcome. 
Table 5 Factors at biopsy influencing renal outcome from ESRD or $50 \%$ drop in eGFR by univariate and multivariate Cox regression

\begin{tabular}{lll}
\hline Risk factors & Univariate Cox Regression & Multivariate Cox Regression \\
\hline & $H R(95 \% \mathrm{Cl})$ & $\mathrm{HR}(95 \% \mathrm{Cl})$
\end{tabular}

Mesangial hypercellularity

$$
\begin{array}{cl}
\text { M0 } & 1 \\
\text { M1 } & 1.8(1.3 \sim 2.3) \\
\text { P-value } & 0.115 \\
\text { Endocapillary } & \text { hypercellularity }
\end{array}
$$

$$
\begin{array}{ll}
\text { E0 } & 1 \\
\text { E1 } & 1.4(0.9 \sim 2.1) \\
\text { P-value } & 0.326
\end{array}
$$

Segmental glomerulosclerosis

$\begin{array}{lll}\text { So } & 1 & 1 \\ \text { S1 } & 3.5(2.3 \sim 5.3) & 2.7(1.8 \sim 4.2) \\ P \text {-value } & <0.001 & <0.001\end{array}$

Tubular atrophy/interstitial fibrosis

$\begin{array}{cll}\text { T0 } & 1 & 1 \\ \text { T1 or T2 } & 2.6(2.1 \sim 3.3) & 6.6(3.9 \sim 11.3) \\ P \text {-value } & <0.001 & <0.001 \\ \text { Crescent } & & 1 \\ \text { C0 } & 1 & 1.8(1.2 \sim 2.5) \\ \text { C1 or C2 } & 2.1(1.5 \sim 2.8) & 0.212 \\ P \text {-value } & <0.001 & \end{array}$

Univariate Cox Regression model: unadjusted. Multivariate Cox Regression model: adjusted for initial eGFR, initial mean arterial pressure, and initial proteinuria

Abbreviations: $\mathrm{Cl}$ Confidence interval, $H R$ Hazard ratio, MO Mesangial hypercellularity $\leq 0.5, M 1$ Mesangial hypercellularity $>0.5$, EO Absence of endocapillary hypercellularity, E1 Presence of endocapillary hypercellularity, So Absence of segmental glomerulosclerosis, S1 Presence of segmental glomerulosclerosis, TO Tubular atrophy/ interstitial fibrosis $0-25 \%$ of cortical area, $T 1$ Tubular atrophy/ interstitial fibrosis $26-50 \%$ of cortical area, $T 2$ Tubular atrophy/interstitial fibrosis $\geq 50 \%$ of cortical area, CO Absence of crescents, C1 Crescents in at least one but $<25 \%$ of glomeruli, C2 Crescents in more than $25 \%$ of glomeruli

Our data showed relevance between lesion S and renal prognosis, which further confirmed that $\mathrm{S}$ was a particular index to judge the prognosis. Many data from the children's cohort have proved the independent predictive value of $\mathrm{S}$ lesion. Children's group from France confirmed that lesion $\mathrm{S}$ was the only histological variable predicting a decline in renal function and was not associated with clinical data at the time of renal biopsy and whether they received immunosuppressive therapy [19]. Studies [20] have revealed S lesion develops from the organization of previous segmental necrotizing or endocapillary inflammatory lesions or in response to podocyte injury and detachment. Therefore, it has also been suggested that in children with active glomerular lesions, special attention needs to be paid to the relationship between lesion $\mathrm{S}$ and $\mathrm{M}$ and $\mathrm{E}$.
The lesion $\mathrm{T}$ was confirmed to be associated with renal failure, which was accord with almost all previous adult validation studies $[4,8,10]$. This may not be surprising because $\mathrm{T}$ lesion represents a more chronic and late stage of IgAN renal damage. However, most children validation studies, such as Japan cohort [15], Sweden cohort [21] and VALIGA cohort [13], failed to confirm that $\mathrm{T}$ lesion could maintain independent predictive value in children. Only the cohort from China by Le et al. [22] and our cohort confirmed that $\mathrm{T}$ lesion has independent predictive value in children population. This difference may be due to only a small proportion of children arrived at a composite terminal during the follow-up in these child studies [13, 15, 21].

In our research, particular interest was given to children with lesion $\mathrm{C}$ as they had a predictive value (HR 2.1, 95\% CI 1.5-2.8) in the univariate analysis, although it did not retain its significance in the multivariate analysis. C lesion was seen in $49 \%$ of the children, however, with $28 \%$ having crescents in $<10 \%$ of glomeruli. At the same time, a higher percentage of children with $C$ were treated with immunosuppression than children without this lesion. Overall, crescents predicted a higher risk of a combined event, although this remained significant only in children not receiving immunosuppression. Thus, crescents in a minority of glomeruli may represent a lesion reversible by immunosuppressive therapy. Our findings suggest that children whose biopsies show these active lesions should be considered for immunosuppressive treatment, which was consistent with a multicenter study [23].

The validation differences among the above different child cohorts are mainly related to the regional and ethnic differences in IgAN, the selection criteria, follow-up time and treatment measures of each study, which emphasizes the need to generate a large database for IgAN children to address the problem of insufficient statistical power due to the small number of progressive cases, especially the relatively short follow-up period.

Our cohort validated the significance of the Oxford classification in a large number of Chinese IgAN children. A comprehensive analysis of the renal pathological features and clinical conditions represented in the cohort suggests that Oxford classification must be considered in conjunction with clinical features (including proteinuria levels and eGFR values) and treatment given after renal biopsy. This also suggests that treatment operations after biopsy may regulate some pathological risk factors. To explore the risk factors and their impact on disease progression by studying the clinical and pathological features of IgAN, the level of diagnosis and treatment of IgAN will ultimately be improved.

The limitations of this study must be recognized. First, retrospective design makes the control of measured 

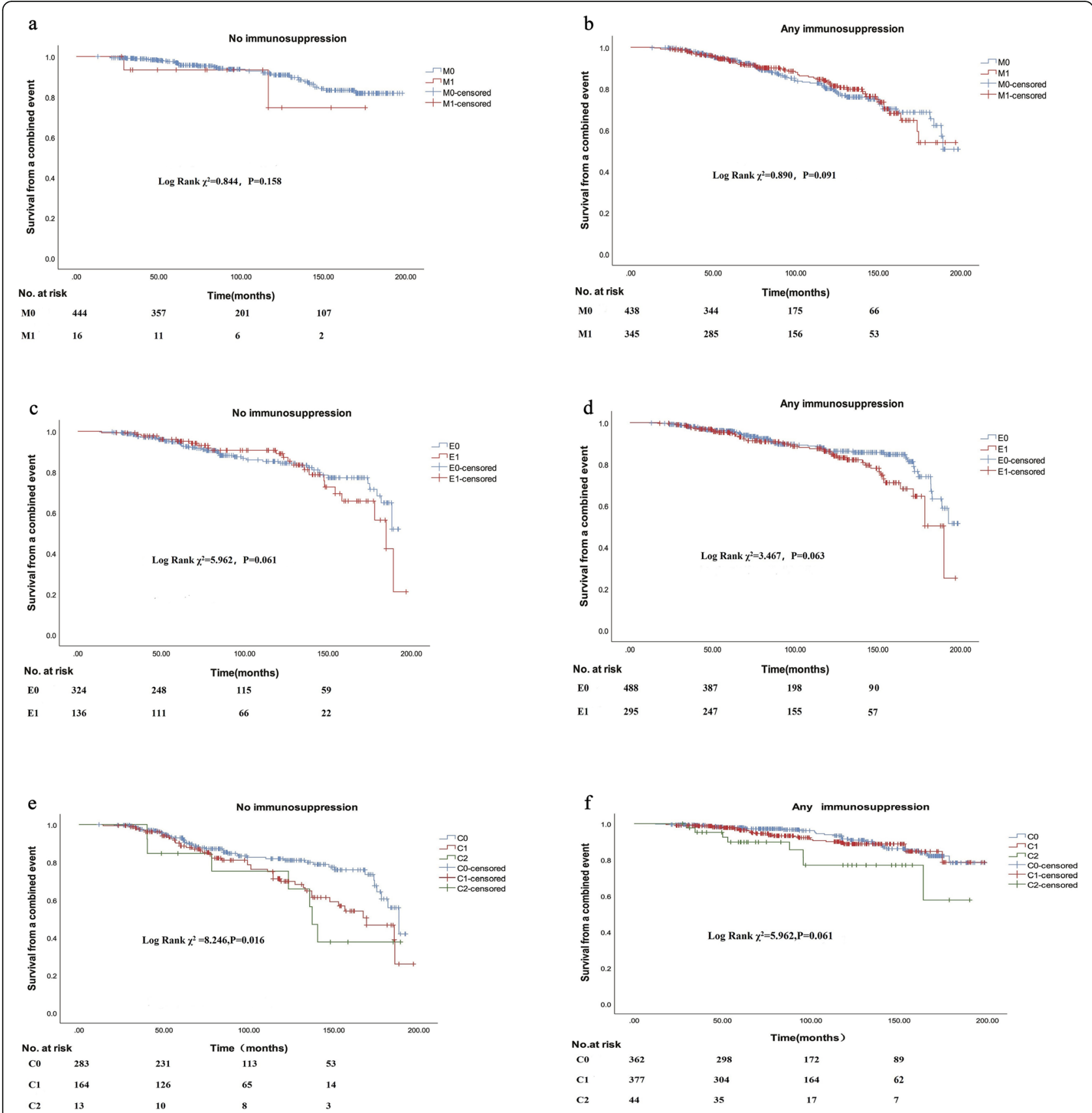

Fig. 3 Predictive value of mesangial hypercellularity, endocapillary hypercellularity and cellular/fibrocellular crescents between immunosuppressive and without immunosuppressive groups. a Effect of the presence of mesangial hypercellularity on survival from a combined event in patients without immunosuppression. $\mathbf{b}$ Effect of the presence of mesangial hypercellularity on survival from a combined event in patients with immunosuppression. $\mathbf{c}$ Effect of the presence of endocapillary hypercellularity on survival from a combined event in patients without immunosuppression. $\mathbf{d}$ Effect of the presence of endocapillary hypercellularity on survival from a combined event in patients with immunosuppression. e Effect of the presence of cellular/ fibrocellular crescents on survival from a combined event in patients without immunosuppression. $\mathbf{f}$ Effect of the presence of cellular/fibrocellular crescents on survival from a combined event in patients with immunosuppression

variables difficult. Second, our results may not be extrapolated to other ethnic groups due to geographical variability in IgAN outcomes. Final, due to the limitations of retrospective studies, not all children were treated with RASB, which may weaken the rigour of the study.
However, some features of this study may increase the strength of these findings, including the broad set of data collected over many years and long-term follow-up by the same team with a well-established clinical protocol, as well as the careful re-evaluation of all renal 
biopsies by two expert pathologist blinded to clinical data and outcome.

\section{Conclusions}

In summary, this study showed that $\mathrm{T}$ and $\mathrm{S}$ lesions were independently linked to poor renal outcome in Chinese IgAN children. In contrast, $C$ lesion showed significant association with prognosis only in children received no immunosuppressive treatment. $M$ and $E$ lesions appeared to be unrelated to renal prognosis.

\section{Abbreviations \\ C: Crescent; Cl: Confidence intervals; eGFR: Estimated glomerular filtration rate; E: Endocapillary proliferation; ESRD: End-stage renal disease; GC: Glucocorticoid; IgAN: IgA Nephropathy; HR: Hazard ratio; IQR: Interquartile range; M: Mesangial proliferation; MAP: Mean arterial pressure; KDIGO: The Kidney Disease Improving Global Outcomes; RASB: Renin angiotensin aldosterone system blockade; S: Segmental sclerosis/adhesion lesion; T: Tubular atrophy/interstitial fibrosis; VALIGA: European Validation Study Of The Oxford Classification Of IgA Nephropathy}

\section{Acknowledgements}

Not applicable.

\section{Authors' contributions}

HW, ZX and CG performed the data collection and analysis and participated in manuscript writing. PZ, XY, RW, MW and YP performed the database setup and statistical analysis. HW, ZX and CG participated in the study design and coordination and helped to draft the manuscript. All of the authors have read and approved the final manuscript

\section{Funding}

The authors acknowledge support from the Clinical Advanced Techniques, Primary Research \& Development Plan of Jiangsu Province (BE2017719) and the Pediatric Medical Innovation Team of Jiangsu Province (CXTDA2017022). The cost of publishing paper was funded in this study. The funders had no role in the design of the study, data collection, analysis and data interpretation, or writing of the manuscript.

\section{Availability of data and materials}

The datasets used and analyzed in this study are available from the first author and corresponding author on reasonable request.

\section{Ethics approval and consent to participate}

The study complied with the Declaration of Helsinki and was approved by the Medical Ethics Committee of Jinling Hospital (Approval number: 2019NZGKJ-266). The committee waived the requirement for informed consent in consideration of the retrospective nature of the study and anonymous data analyses.

\section{Consent for publication}

Not applicable.

\section{Competing interests}

The authors declare that they have no competing interests.

\section{Author details}

${ }^{1}$ Department of Pediatrics, Jinling Hospital, The First School of Clinical Medicine, Southern Medical University, Nanjing, China. ${ }^{2}$ Department of Pediatrics, Nanfang Hospital, Southern Medical University, Guangzhou, China. ${ }^{3}$ Department of Pediatrics, Jinling Hospital, Nanjing Medical University, Nanjing, China.
Received: 19 August 2019 Accepted: 26 June 2020

Published online: 01 July 2020

\section{References}

1. Suzuki H. IgA nephropathy. Nihon Jinzo Gakkai Shi. 2015;57(8):1349-53.

2. Canetta PA, Kiryluk K, Appel GB. Glomerular diseases: emerging tests and therapies for IgA nephropathy. Clin J Am Soc Nephrol. 2014;9(3):617-25.

3. Roberts IS. Oxford classification of immunoglobulin a nephropathy: an update. Curr Opin Nephrol Hypertens. 2013;22(3):281-6.

4. Trimarchi H, Barratt J, Cattran DC, Cook HT, Coppo R, Haas M, Liu ZH, Roberts IS, Yuzawa Y, Zhang H, Feehally J. IgAN classification working Group of the International IgA nephropathy network and the Renal Pathology Society; conference participants. Oxford classification of IgA nephropathy 2016: an update from the IgA nephropathy classification working group. Kidney Int. 2017:91(5):1014-21.

5. Schwartz GJ, Schneider MF, Maier PS, Moxey-Mims M, Dharnidharka VR, Warady BA, Furth SL, Muñoz A. Improved equations estimating GFR in children with chronic kidney disease using an immunonephelometric determination of cystatin C. Kidney Int. 2012;82(4):445-53.

6. Selistre L, Rabilloud M, Cochat P, de Souza V, Iwaz J, Lemoine S, Beyerle F, Poli-de-Figueiredo CE, Dubourg L. Comparison of the Schwartz and CKD-EPI equations for estimating glomerular filtration rate in children, adolescents, and adults: a retrospective cross-sectional study. PLoS Med. 2016;13(3): e1001979.

7. Ku E, Sarnak MJ, Toto R, McCulloch CE, Lin F, Smogorzewski M, Hsu CY. Effect of blood pressure control on long-term risk of end-stage renal disease and death among subgroups of patients with chronic kidney disease. J Am Heart Assoc. 2019:8(16):e012749.

8. Beck L, Bomback AS, Choi MJ, Holzman LB, Langford C, Mariani LH, Somers MJ, Trachtman H, Waldman M. KDOQI US commentary on the 2012 KDIGO clinical practice guideline for glomerulonephritis. Am J Kidney Dis. 2013; 62(3):403-41.

9. Bellur SS, Lepeytre F, Vorobyeva O, Troyanov S, Cook HT, Roberts IS. International IgA nephropathy working group. Evidence from the Oxford classification cohort supports the clinical value of subclassification of focal segmental glomerulosclerosis in IgA nephropathy. Kidney Int. 2017;91(1): 235-43.

10. Zhang L, Li J, Yang S, Huang N, Zhou Q, Yang Q, Yu X. Clinicopathological features and risk factors analysis of IgA nephropathy associated with acute kidney injury. Ren Fail. 2016;38(5):799-805.

11. Nasri H, Mubarak M. Extracapillary proliferation in IgA nephropathy; recent findings and new ideas. J Nephropathol. 2015;4(1):1-5.

12. Rodrigues JC, Haas M, Reich HN. IgA nephropathy. Clin J Am Soc Nephrol. 2017:12(4):677-86.

13. Coppo R, Troyanov S, Bellur S, Cattran D, Cook HT, Feehally J, Roberts IS, Morando L, Camilla R, Tesar V, Lunberg S, Gesualdo L, Emma F, Rollino C, Amore A, Praga M, Feriozzi S, Segoloni G, Pani A, Cancarini G, Durlik M, Moggia E, Mazzucco G, Giannakakis C, Honsova E, Sundelin BB, Di Palma AM, Ferrario F, Gutierrez E, Asunis AM, Barratt J, Tardanico R, PerkowskaPtasinska A, VALIGA study of the ERA-EDTA Immunonephrology Working Group. Validation of the Oxford classification of IgA nephropathy in cohorts with different presentations and treatments. Kidney Int. 2014:86(4):828-36.

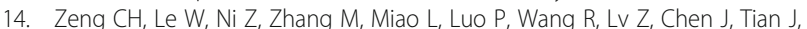
Chen N, Pan X, Fu P, Hu Z, Wang L, Fan Q, Zheng H, Zhang D, Wang Y, Huo $Y$, Lin $H$, Chen S, Sun S, Wang Y, Liu Z, Liu D, Ma L, Pan T, Zhang A, Jiang $X$, Xing C, Sun B, Zhou Q, Tang W, Liu F, Liu Y, Liang S, Xu F, Huang Q, Shen H, Wang J, Shyr Y, Phillips S, Troyanov S, Fogo A, Liu ZH. A multicenter application and evaluation of the oxford classification of IgA nephropathy in adult Chinese patients. Am J Kidney Dis. 2012:60(5):812-20.

15. Shima Y, Nakanishi K, Kamei K, Togawa H, Nozu K, Tanaka R, Sasaki S, lijima K, Yoshikawa N. Disappearance of glomerular IgA deposits in childhood IgA nephropathy showing diffuse mesangial proliferation after 2 years of combination/prednisolone therapy. Nephrol Dial Transplant. 2011;26(1):163-9.

16. Working Group of the International IgA Nephropathy Network and the Renal Pathology Society, Cattran DC, Coppo R, Cook HT, Feehally J, Roberts IS, Troyanov S, Alpers CE, Amore A, Barratt J, Berthoux F, Bonsib S, Bruijn JA, D'Agati V, D'Amico G, Emancipator S, Emma F, Ferrario F, Fervenza FC, Florquin S, Fogo A, Geddes CC, Groene HJ, Haas M, Herzenberg AM, Hill PA, Hogg RJ, Hsu SI, Jennette JC, Joh K, Julian BA, Kawamura T, Lai FM, Leung CB, Li LS, Li PK, Liu ZH, Mackinnon B, Mezzano S, Schena FP, Tomino Y, Walker PD, Wang H, Weening JJ, Yoshikawa N, Zhang H. The Oxford 
classification of IgA nephropathy: rationale, clinicopathological correlations, and classification. Kidney Int. 2009;76(5):534-45.

17. El Karoui K, Hill GS, Karras A, Jacquot C, Moulonguet L, Kourilsky O, Frémeaux-Bacchi V, Delahousse M, Duong Van Huyen JP, Loupy A, Bruneval P, Nochy D. A clinicopathologic study of thrombotic microangiopathy in IgA nephropathy. J Am Soc Nephrol. 2012;23(1):137-48.

18. Chakera A, MacEwen C, Bellur SS, Chompuk LO, Lunn D, Roberts ISD. Prognostic value of endocapillary hypercellularity in IgA nephropathy patients with no immunosuppression. J Nephrol. 2016;29(3):367-75.

19. Cambier A, Rabant M, Peuchmaur M, Hertig A, Deschenes G, Couchoud C, Kolko A, Salomon R, Hogan J, Robert T. Immunosuppressive treatment in children with IgA nephropathy and the clinical value of Podocytopathic features. Kidney Int Rep. 2018;3(4):916-25.

20. Trimarchi H, Coppo R. Podocytopathy in the mesangial proliferative immunoglobulin a nephropathy: new insights into the mechanisms of damage and progression. Nephrol Dial Transplant. 2019;34(8):1280-5.

21. Edström Halling S, Söderberg MP, Berg UB. Predictors of outcome in paediatric IgA nephropathy with regard to clinical and histopathological variables (Oxford classification). Nephrol Dial Transplant. 2012;27(2):715-22.

22. Le W, Zeng CH, Liu Z, Liu D, Yang Q, Lin RX, Xia ZK, Fan ZM, Zhu G, Wu Y, Xu H, Zhai Y, Ding Y, Yang $X$, Liang $S$, Chen $H, X u F$, Huang $Q$, Shen $H$, Wang J, Fogo AB, Liu ZH. Validation of the Oxford classification of IgA nephropathy for pediatric patients from China. BMC Nephrol. 2012;13:158.

23. Haas M, Verhave JC, Liu ZH, Alpers CE, Barratt J, Becker JU, Cattran D, Cook HT, Coppo R, Feehally J, Pani A, Perkowska-Ptasinska A, Roberts IS, Soares MF, Trimarchi H, Wang S, Yuzawa Y, Zhang H, Troyanov S, Katafuchi R. A multicenter study of the predictive value of crescents in IgA nephropathy. J Am Soc Nephrol. 2017;28(2):691-701.

\section{Publisher's Note}

Springer Nature remains neutral with regard to jurisdictional claims in published maps and institutional affiliations.

Ready to submit your research? Choose BMC and benefit from:

- fast, convenient online submission

- thorough peer review by experienced researchers in your field

- rapid publication on acceptance

- support for research data, including large and complex data types

- gold Open Access which fosters wider collaboration and increased citations

- maximum visibility for your research: over $100 \mathrm{M}$ website views per year

At $\mathrm{BMC}$, research is always in progress.

Learn more biomedcentral.com/submissions 